\title{
Curcumin induces the apoptosis of A549 cells via oxidative stress and MAPK signaling pathways
}

\author{
QINGHUA YAO ${ }^{1}$, MIAO LIN $^{2}$, YUQI WANG ${ }^{2}$, YUEBIAO LAI $^{2}$, JINGJING HU $^{2}$, TING FU $^{2}$, \\ LU WANG $^{2}$, SHUYUAN LIN ${ }^{2}$, LIANGLIANG CHEN ${ }^{3}$ and YONG GUO ${ }^{3}$ \\ ${ }^{1}$ Key Laboratory of Traditional Chinese Medicine Oncology, Zhejiang Cancer Hospital, Banshan Qiao, \\ Hangzhou, Zhejiang 310022; ${ }^{2}$ Zhejiang Chinese Medical University, Hangzhou, Zhejiang 310053; \\ ${ }^{3}$ The First Affiliated Hospital of Zhejiang Chinese Medical University, Hangzhou, Zhejiang 310006, P.R. China
}

Received December 10, 2014; Accepted August 10, 2015

DOI: $10.3892 /$ ijmm.2015.2327

\begin{abstract}
Curcumin has been found to exhibit anticancer activity and certain studies have shown that curcumin triggers the apoptosis of human A549 lung adenocarcinoma cells. However, the mechanism underlying curcumin-mediated apoptosis is not completely understood. The present study was designed to investigate the effect of curcumin on the induction of apoptosis and apoptosis-related factors in human A549 lung adenocarcinoma cells. Treatment of A549 cells with curcumin caused a concentration-dependent inhibition of cell growth and an increase in apoptosis, as confirmed by THE MTT assay, flow cytometry and morphology analysis. Curcumin-treatment of A549 cells induced a loss of the mitochondrial membrane potential and increased cytosolic cytochrome $c$. Furthermore, curcumin-induced apoptosis was accompanied by changes in intracellular oxidative stress-related enzymes, including decreased intracellular reactive oxygen species levels, increased superoxide dismutase and decreased malondialdehyde and 4-hydroxynonenal. In addition, induction of apoptosis was also accompanied by phosphorylation and activation of mitogen-activated protein kinase signaling pathway factors c-Jun N-terminal kinase, p38 and extracellular signal-regulated kinase.
\end{abstract}

\section{Introduction}

Lung cancer is the main leading cause of cancer-related fatality worldwide, with a rapidly increasing rate in China and other Asian countries (1-4). Chemotherapy is currently the most frequently used treatment for lung cancer and other types of cancer. However, while this method of treatment kills cancer

Correspondence to: Dr Yong Guo, The First Affiliated Hospital of Zhejiang Chinese Medical University, 54 Youdian Road, Hangzhou, Zhejiang 310006, P.R. China

E-mail: guoyong1047@163.com

Key words: curcumin, apoptosis, mitochondrial signaling pathway, MAPK pathway, oxidative stress cells, it also destroys some normal cells. Thus, the identification of novel natural compounds with low toxicity and high selectivity for killing cancer cells is a significant area in cancer research (5), and several natural products have been used as alternative treatments for cancer $(6,7)$.

Curcumin, a natural and crystalline compound isolated from the plant Curcuma longa, has been widely studied for its anti-inflammatory, antiangiogenic, antioxidant and anticancer effects in Chinese systems of medicine $(8,9)$. Additionally, previous studies have shown that curcumin exhibits antiproliferative and anticarcinogenic properties in a wide variety of cell lines and animals (10,11). Previous studies have demonstrated that curcumin inhibits the growth and apoptosis of human A549 lung adenocarcinoma cells (12-14). However, the mechanisms of curcumin-induced apoptosis via oxidative stress remain unclear.

The physiological status of the reactive oxygen species (ROS) levels can regulate cell proliferation: When intracellular ROS levels are above a certain threshold, ROS inhibit the cell cycle, leading to increased cell apoptosis and necrosis. In the tumor cells it often maintains a higher state of oxidation, with higher levels of oxygen free radicals and lower levels of the antioxidant enzyme activity. This higher oxidation state can activate certain transcription factors and associated genes, such as $N F-\kappa B$ and $A P I$, thus, ensuring the survival, proliferation and migration of tumor cells. Certain research has shown that the antitumor function of curcumin occurs by regulation of the intracellular redox state $(15,16)$. In tumor cells, low concentrations of curcumin inhibit cell proliferation, with no induction of apoptosis. However, intracellular ROS are slightly decreased and the level of superoxide dismutase (SOD) is elevated, which causes lipid peroxidation in the presence of other factors to suppress malondialdehyde (MDA) and 4-hydroxynonenal (4-HNE). In high concentrations of curcumin, cellular ROS declines rapidly and the SOD level increases, along with a significant increase of apoptosis products $(17,18)$.

Heat-shock proteins (HSP) are necessary proteins under physiological conditions that are upregulated in response to stress (particularly heat stress). Higher expression of HSP70 was identified in tumor cells compared with normal cells and was closely associated with histological types of lung cancer 
and prognosis (19). High expression of HSP70 may be required to maintain the stability of tumor cells through regulation of gene expression and immune response (20). Under conditions of oxidative stress, ionizing radiation and heat-shock stress, HSP70 expression increases, and subsequently participates in the process of the B-cell lymphoma 2 (Bcl-2) family to regulate tumor cell apoptosis (21). HSP70, as a molecular chaperone, changes the conformation of the $\mathrm{Bcl}-2$-associated $\mathrm{X}$ protein (Bax) gene to prevent cellular apoptosis. Upon exposure to HSP70 inhibitors, the tumor cells undergo apoptosis (22).

These findings suggest that HSPs are closely associated with ROS in the process of tumor cell apoptosis. Following the disruption of the high expression level of ROS and HSP70 in the tumor cells, the cells undergo apoptosis. Although numerous studies have confirmed that curcumin induces apoptosis via the mitochondrial pathway $(23,24)$, the signaling pathways for the ROS-mediated mitochondrial apoptotic cell death triggered by curcumin still remain unclear, particularly in lung cancer cells.

In the present study, the molecular mechanisms of the effects of curcumin on the induction of apoptosis of the human A549 non-small cell lung cancer cell line were examined. The data indicate that the initiation of curcumin-induced apoptotic signaling involves decreased ROS, HSP70 and mitochondrial membrane potential (MMP). These findings aid in elucidating the mechanisms of curcumin-induced apoptosis and may contribute to the development of a novel drug based on curcumin alone or in combination therapies.

\section{Materials and methods}

Reagents and cell lines. Curcumin $\left(\mathrm{C}_{21} \mathrm{H}_{20} \mathrm{O}_{6}\right)$ was purchased from Sigma Chemical (St. Louis, MO, USA). Propidium iodide (PI) and Annexin V-fluorescein isothiocyanate (FITC) were purchased from BD Pharmingen (Minneapolis, MN, USA). HSP70, Bcl-2, Bax, caspase-3, caspase-9, cleaved caspase-3, cleaved caspase-9, total c-Jun N-terminal kinase (JNK), p38 and extracellular signal-regulated kinase (ERK), and phosphorylated JNK, p38 and ERK were purchased from Cell Signaling Technology (Danvers, MA, USA). U0126 (ERK inhibitor), SB203580 (p38 inhibitor) and SP600125 (JNK inhibitor) were from Sigma Chemical. Dichlorodihydro-fluorescein diacetate (DCFH-DA) was obtained from Beyotime Institute of Biotechnology (Jiangsu, China). MDA and 4-HNE were obtained from Nanjing Jiancheng Bioengineering Institute (Jiangsu, China). MitoTracker was obtained from Molecular Probes (Eugene, OR, USA). Fetal bovine serum (FBS), RPMI-1640 and penicillin-streptomycin were obtained from Thermo Fisher Scientific (Waltham, MA, USA). A549 human lung carcinoma cells were obtained from the Shanghai Cell Center of Chinese Academy of Sciences.

Cell culture. The A549 cell line was cultured in RPMI-1640 supplemented with $10 \%$ FBS and was grown in a humidified atmosphere with $5 \% \mathrm{CO}_{2}$ at $37^{\circ} \mathrm{C}$.

Cell growth inhibition assay. Cell proliferation was measured by cell counting and MTT assays. Cells were seeded in 96-well plates with $2 \times 10^{4}$ cells/well. After $24 \mathrm{~h}$, the cells were incubated with different concentrations of curcumin for different times. Following incubation, MTT solution at $20 \mu \mathrm{l} /$ well [5 $\mathrm{mg} / \mathrm{ml}$ in phosphate-buffered saline (PBS)] was added and cells were incubated for an additional $5 \mathrm{~h}$. The medium was aspirated and replaced with $150 \mu \mathrm{l} /$ well dimethyl sulfoxide (DMSO) to dissolve the formazan salt. The color intensity of the formazan solution, which reflects the cell growth condition, was measured at $570 \mathrm{~nm}$ using a microplate reader (BioTek Instruments, Winooski, VT, USA). In the presence of antioxidants, the cells were washed with PBS and evaluated by the MTT assay.

Flow cytometry. For detection of apoptosis by fluorescence-activated cell sorting, $\sim 25 \times 10^{4}$ cells $/ \mathrm{ml}$ in $6-\mathrm{cm}$ plates were treated with various concentrations of curcumin. The cells were harvested and used for FITC-conjugated Annexin V and PI staining for $5 \mathrm{~min}$ at room temperature. The stained cells were analyzed by flow cytometry (BD Biosciences, Minneapolis, MN, USA) to determine the percentages of apoptotic cells.

Electron microscopy. A549 cells were trypsinized and fixed in ice-cold $2.5 \%$ electron microscopy grade glutaraldehyde in PBS (pH 7.3). The specimens were rinsed with PBS, post-fixed in $1 \%$ osmium tetroxide with $0.1 \%$ potassium ferricyanide, dehydrated through a graded series of ethanol (30-90\%), and embedded in Epon. Semi-thin (300 nm) sections were cut using a Reichart Ultracut, stained with $0.5 \%$ toluidine blue, and examined under a light microscope (Olympus, Tokyo, Japan). Ultra-thin sections $(65 \mathrm{~nm})$ were stained with $2 \%$ uranyl acetate and Reynold's lead citrate, and examined on a transmission electron microscope (magnification, $x 5,000$ ).

Measurement of MMP. A549 cells were seeded in 6-well plates with $30 \times 10^{4}$ cells/well. After 24 -h incubation, cells were treated with curcumin $(20 \mu \mathrm{M})$. After $24 \mathrm{~h}$, cells were incubated with $5 \mu \mathrm{M} \mathrm{JC}-1$ fluorescent dye for $30 \mathrm{~min}$ at $37^{\circ} \mathrm{C}$ in the dark, and were subsequently washed with PBS. MMP was evaluated qualitatively under a fluorescence microscope (Olympus IX81) using a $568 \mathrm{~nm}$ filter.

Immunofluorescence analysis. Cells were incubated with $10 \mathrm{nM}$ MitoTracker Green for $1 \mathrm{~h}$. Cells were subsequently cyto-spinned on glass slides, washed twice with PBS, fixed with $4 \%$ paraformaldehyde for $10 \mathrm{~min}$, permeabilized with $0.3 \%$ Triton for 10 min and incubated with PBS containing $10 \%$ bovine serum albumin (BSA) for $1 \mathrm{~h}$ at room temperature. Primary antibodies diluted in PBS containing 1\% BSA (1/200 for anti-cytochrome $c$ antibody; Cell Signaling Technology) were incubated with cells at $4^{\circ} \mathrm{C}$ overnight. Cells were washed and incubated with the appropriate fluorescent secondary antibody. Cells were washed twice in PBS and stained with 4',6-diamidino-2-phenylindole. Fluorescent images were obtained on a Zeiss Axioplan microscope (Carl Zeiss AG, Oberkochen, Germany) and 10 sections of each preparation were scanned.

Determination of ROS, SOD, MDA and 4-HNE levels. The effects of curcumin on intracellular ROS generation were evaluated by DCFH-DA fluorescence assay. In brief, A549 cells were co-incubated with different concentrations of curcumin for various times, and were subsequently 
incubated with DCFH-DA at a final concentration of $10 \mu \mathrm{M}$ at $37^{\circ} \mathrm{C}$ for $30 \mathrm{~min}$. Cells were harvested, washed three times with PBS and were suspended in PBS $\left(1 \times 10^{6}\right.$ cells $\left./ \mathrm{ml}\right)$, and the fluorescence intensity was measured on a fluorescence reader (BD Biosciences, Franklin Lakes, NJ, USA). Relative DCF fluorescence intensity of treated cells was expressed as percentage of the control (as 100\%).

SOD activity was determined by specific assay kits purchased from Beyotime Institute of Biotechnology, according to the manufacturer's instructions. In brief, following curcumin treatment, cells were lysed in cell lysis buffer. Cell lysates $(100 \mu \mathrm{g} / w e l l)$ were evaluated for SOD activity, and SOD activity was expressed as a percentage of the control.

MDA activity was measured by the assay kit (Nanjing Jiancheng Bioengineering Institute) and performed according to the manufacturer's instructions. Briefly, curcumin-treated cells were harvested and lysed in cell lysis buffer and MDA activity was evaluated. MDA activity was determined by comparison with the standard curve prepared from predetermined MDA standards.

4-HNE, a biomarker of oxidative stress, was examined using an ELISA kit (Nanjing Jiancheng Bioengineering Institute). Briefly, 4-HNE protein adducts present in the sample or standard were probed with the 4-HNE primary antibody, followed by incubation with a horseradish peroxidase-conjugated secondary antibody. The 4-HNE protein adduct content in the unknown sample was determined by comparison with the standard curve prepared from predetermined HNE-BSA standards.

Western blot analysis. Cells were seeded in $60-\mathrm{mm}$ dishes and incubated with different concentrations of curcumin for different times. Proteins were extracted in lysis buffer, supplemented with $1 \mathrm{mM}$ phenylmethylsulfonyl fluoride, protease inhibitor and phosphatase inhibitors. Total proteins $(25 \mu \mathrm{g})$ were electrophoresed on $10 \%$ polyacrylamide gels and transferred to nitrocellulose membranes. Membranes were blocked with 5\% dried skimmed milk in Tris-buffered saline/Tween-20 buffer for $1 \mathrm{~h}$ at room temperature. The membranes were incubated with primary antibodies at 1:1,000 dilution in $5 \%$ non-fat milk overnight at $4^{\circ} \mathrm{C}$, and secondary antibodies $(1: 5,000)$ were subsequently incubated for $2 \mathrm{~h}$ at room temperature. Protein bands were visualized with the enhanced chemiluminescence method. The results were corrected for protein loading by normalization for $\beta$-actin expression and quantified by Quantity One software (Bio-Rad Laboratories, Inc., Hercules, CA, USA).

Statistical analysis. All data are presented as mean \pm standard deviation. Statistical analysis of the results was performed by one-way analysis of variance followed by the SPSS statistical package (SPSS 13.0 for Windows; SPSS, Inc., Chicago, IL, USA). For all the statistical analyses, $\mathrm{P}<0.05$ or $\mathrm{P}<0.01$ were considered to indicate a statistically significant difference.

\section{Results}

Curcumin inhibits cell growth of A549 cells. To evaluate the growth inhibitory effects of curcumin, A549 cells were treated with curcumin for various times and viable cells were measured by the MTT assay. A decrease in cell viability was observed in response to curcumin treatment in a time- and dose-dependent manner (Fig. 1A).

Curcumin induces the apoptosis of A549 cells. To determine whether the growth inhibition by curcumin was associated with apoptosis, the degree of apoptosis was examined by PI and Annexin V staining through flow cytometric analysis. As shown in Fig. 1B and C, curcumin caused a significant inhibition of cell proliferation in a concentration-dependent manner. When cells were treated with curcumin $(5-40 \mu \mathrm{M})$ for $24 \mathrm{~h}$, the proportion of $\mathrm{AV}^{+} / \mathrm{PI}^{-}$(apoptotic cells) was increased from $1.65 \%$ of control cells to $26.58 \%$ of the group treated with $20 \mu \mathrm{M}$ curcumin $(\mathrm{P}<0.05)$, the $40 \mu \mathrm{M}$ curcumin group was increased by $\sim 30.63 \%(\mathrm{P}<0.05)$. In addition, the percentage of apoptotic cells increased in a time- and dose-dependent manner. As shown in Fig. 1D, no clear difference in the number of $\mathrm{AV}^{+} / \mathrm{PI}$ (apoptotic cells) was observed under relatively short incubation conditions ( 3 and $6 \mathrm{~h}$ ). After 12-h treatment, however, the proportion of apoptotic cells slowly increased. Apoptotic cells were significantly elevated in $20 \mu \mathrm{M}$ curcumin-treated cells, from $5.7 \%$ in untreated cells to $29.06 \%$ after 24 -h treatment. These results suggest that curcumin-induced apoptosis has a dose- and time-dependent association.

Involvement of caspase activation in the curcumin-induced apoptotic effect. Caspase activation is a significant event in the proteolytic cascade elicited by apoptotic stimuli, particularly caspase- 3 , which is an effector caspase that has a critical role in cell death induced by a variety of stimuli. To determine the mechanism by which curcumin treatment trigger A549 cell apoptosis, the protein levels of cleaved caspase- 9 and -3 were examined by western blot analyses. The result suggested that caspase- 9 and -3 were activated in a dose-dependent manner in curcumin-treated cells (Fig. 2).

Curcumin causes changes to the morphological features of apoptosis. The morphological characteristics of A549 cells treated with curcumin were further examined by electron microscopy. As shown in Fig. 3, the control cells exhibited an intact nuclear structure, while cells treated with curcumin showed morphological changes that are characteristic of apoptosis, including disappearance of mitochondrial cristae, cell shrinkage, chromatin aggregation containing a half-moon of condensed chromatin, and appearance of membrane blebbing and numerous apoptotic bodies.

Curcumin induces cytochrome c translocation. Cytochrome $c$ release from mitochondria is a critical event promoting the intrinsic death pathway by apoptotic protease activating factor 1 (Apaf-1)-mediated caspase-3 activation and apoptosis. The effects of curcumin on cytochrome $c$ translocation were subsequently examined. In DMSO-treated control A549 cells, cytochrome $c$ exhibited punctate cytoplasmic staining in agreement with its localization in mitochondria, as evidenced by yellow-orange staining from merging with MitoTracker Green staining (Fig. 4A). Upon treatment with curcumin, the yellow-orange staining was markedly abolished. A large fraction of curcumin-treated cells exhibited red fluorescence, indicating cytochrome $c$ release from the mitochondria into 

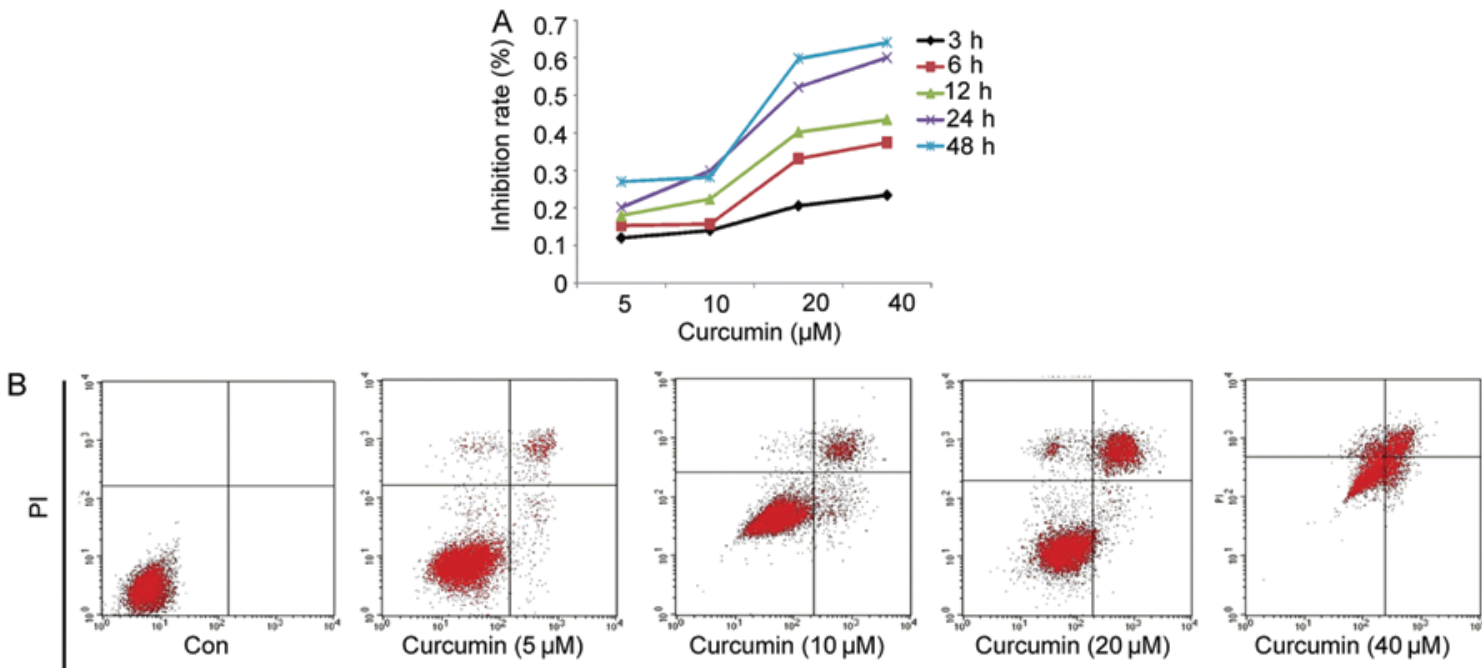

Annexin V
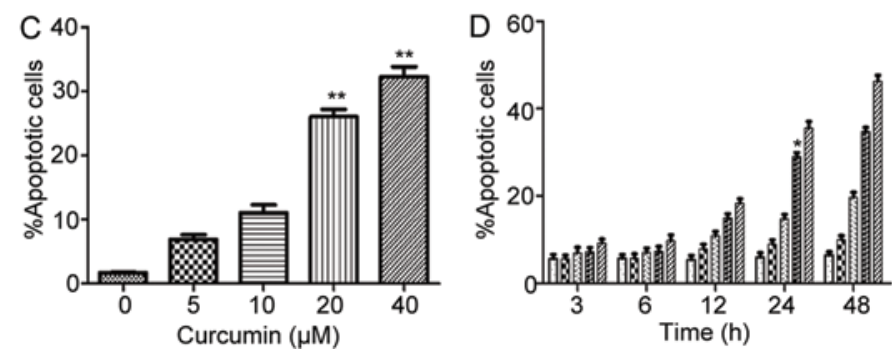

$\square 0$

$\times 5$

붕 10

20

40

Figure 1. Effects of curcumin on the proliferation and apoptosis of A549 cells. (A) MTT assay evaluation of the inhibition of proliferation of A549 cells incubated with curcumin at different concentrations and different times. (B) Flow cytometry of Annexin V and PI double staining of A549 cells treated with curcumin at different concentrations for $24 \mathrm{~h}$. (C) Columns show the percentages of apoptotic A549 cells incubated with curcumin corresponding to (A). Significant differences were observed between 0 to $20 \mu \mathrm{M}$, and 0 to $40 \mu \mathrm{M}\left({ }^{* *} \mathrm{P}<0.05\right)$. (D) Curcumin induction of cell apoptosis was in a dose- and timedependent manner. Significant differences were observed at $20 \mu \mathrm{M}$ and $24 \mathrm{~h}(\mathrm{P}<0.05)$.

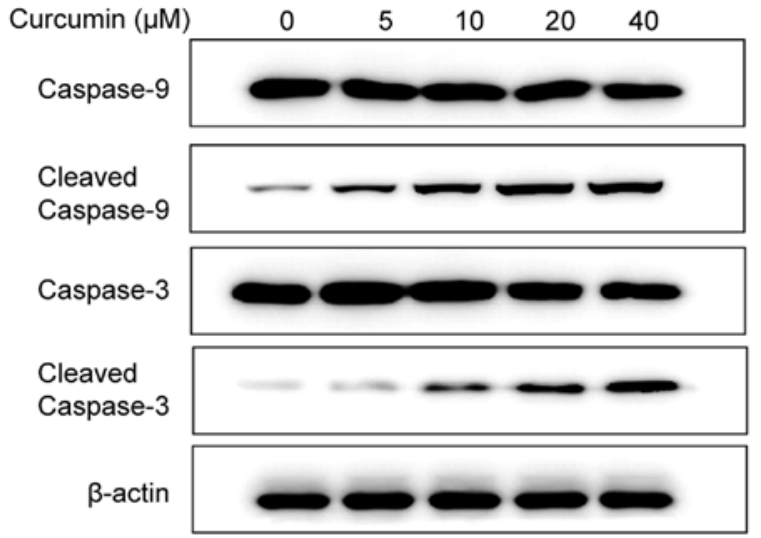

Figure 2. Effects of curcumin on caspase activation. A549 cells were treated with curcumin $(5-40 \mu \mathrm{M})$ for $24 \mathrm{~h}$, and were lysed and analyzed by western blot analysis.

the cytosol (Fig. 4B). These data suggested that curcumin treatment of A549 cells increased the level of cytochrome $c$ in the cytosol with a concomitant decreased level of cytochrome $c$ in the mitochondria.

Effects of curcumin on MMP. To further confirm the observations regarding the induction of apoptosis of A549 cells by curcumin, A549 cells were exposed to 5-40 $\mu \mathrm{M}$ curcumin for
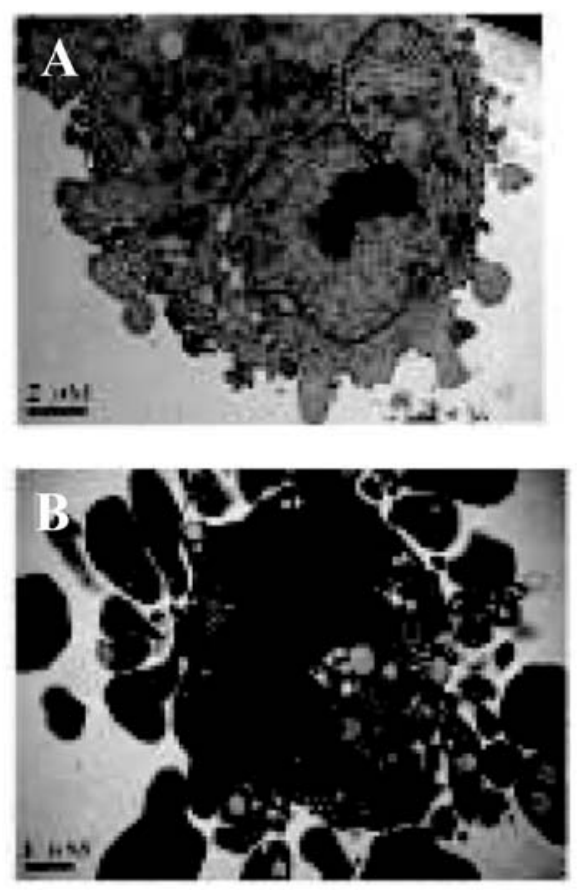

Figure 3. Effect of curcumin on the cellular ultrastructure. Cells were treated with $20 \mu \mathrm{M}$ curcumin for $24 \mathrm{~h}$ and fixed with $2 \%$ glutaraldehyde for $15 \mathrm{~min}$. Following several rinses in phosphate-buffered saline, cells were post-fixed and dehydrated as described in Materials and methods. (A) Control cells exposed to $0.1 \%$ dimethyl sulfoxide. (B) A549 cells exposed to curcumin $(20 \mu \mathrm{M})$. 

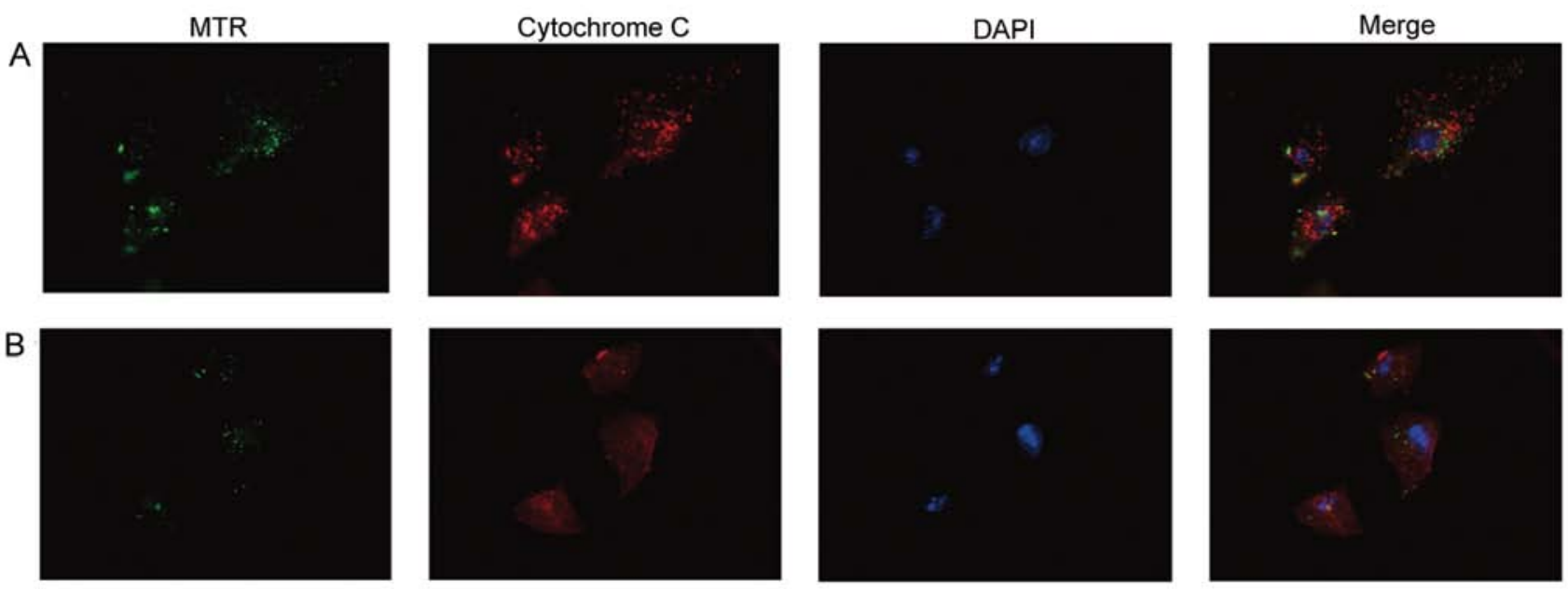

Figure 4. Curcumin-induced cytochrome $c$ translocation. A549 cells were incubated for $24 \mathrm{~h}$ with (A) dimethyl sulfoxide or (B) $20 \mu \mathrm{M}$ curcumin. Two groups were incubated for $1 \mathrm{~h}$ with $10 \mathrm{~nm}$ MitoTracker (green), and subsequently cytospun on glass slides, fixed and stained with cytochrome $c$ antibody (red). The slides were analyzed by a confocal laser scanning microscope. MTR, MitoTracker Red; DAPI, 4',6-diamidino-2-phenylindole.
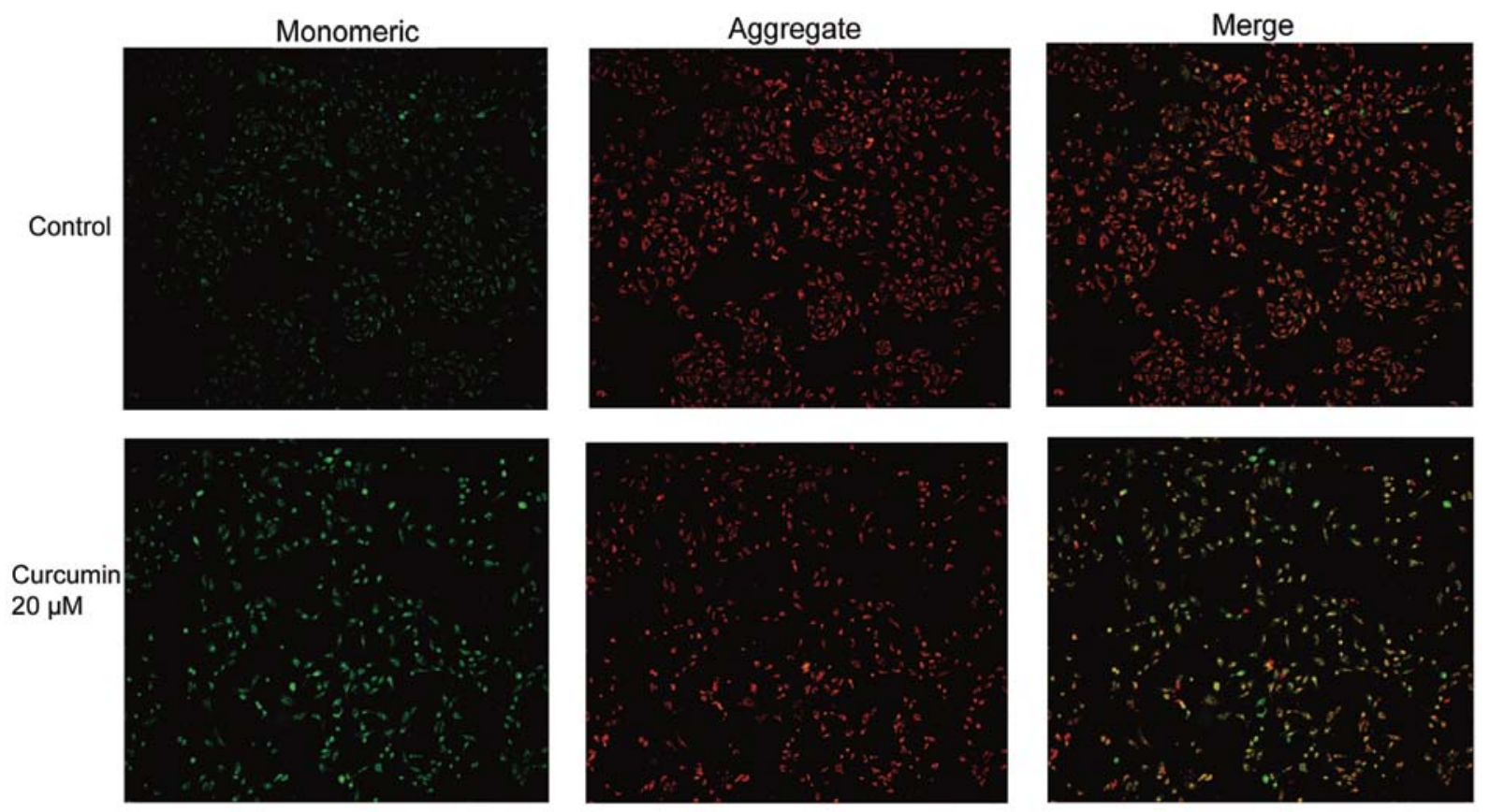

Figure 5. Effects of curcumin on matrix metalloproteinase. A549 cells were treated with $20 \mu \mathrm{M}$ curcumin for $24 \mathrm{~h}$. Cells were stained with $10 \mu \mathrm{g} / \mathrm{ml}$ of JC- 1 and observed under a confocal microscope.

$24 \mathrm{~h}$ and were subsequently stained with $\mathrm{JC}-1$, which is used to assess the integrity of the MMP. Cellular mitochondria are stained red when their membranes are intact and polarized, and exhibit green fluorescence when the membranes are depolarized. Fluorescence microscopic evaluation of JC-1-stained control cells showed heterogeneous staining of the cytoplasm with red and green fluorescence coexisting in the same cell (Fig. 5). This staining was consistent with mitochondrial localization. In the control group, the red fluorescence was distributed throughout the cytoplasm. Cells treated with curcumin showed marked changes in MMP, such as the disappearance of red fluorescence and increase of green fluorescence in the majority of cells. These data indicate that curcumin induces loss of MMP.
Effect of curcumin on the expression of the Bcl-2 family of proteins. To further investigate the mechanisms underlying curcumin-induced apoptosis in lung cancer cells, the apoptotic proteins Bcl-2 and Bax were analyzed by western blot analysis. As shown in Fig. 6A, the proapoptotic protein Bax was increased following treatment with curcumin for $24 \mathrm{~h}$, whereas antiapoptotic protein Bcl-2 levels decreased. Therefore, an increase in the $\mathrm{Bax} / \mathrm{Bcl}-2$ ratio may be involved in apoptosis induced by curcumin (Fig. 6B).

Effects of curcumin on the levels of intracellular ROS, SOD, $M D A$ and 4-HNE. ROS are important signaling molecules that have a role in gene expression, proliferation and apoptosis, as well as oxygen sensing in various cell types. To explore the 

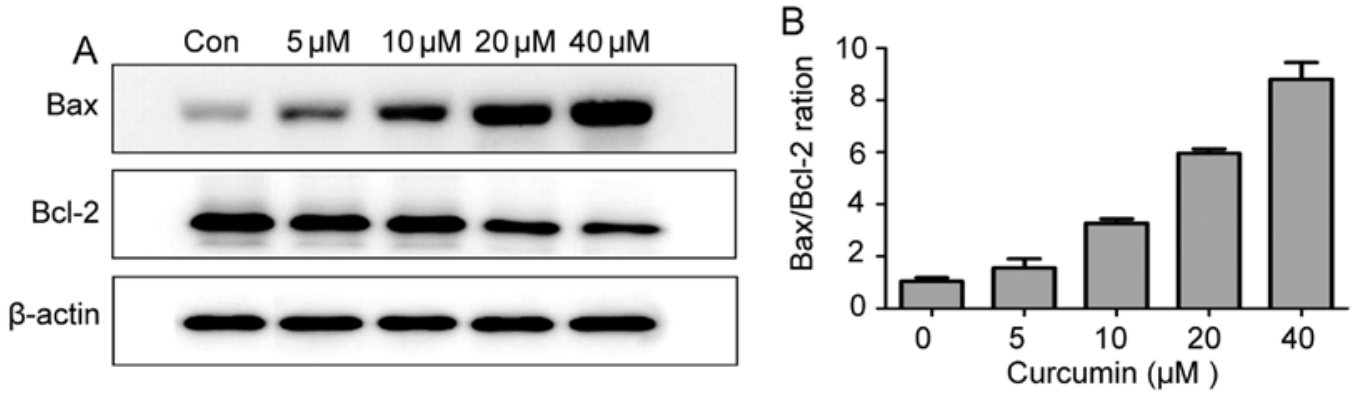

Figure 6. Effect of curcumin on the expression of the Bcl-2 family proteins in A549 cells. (A) Cells were incubated in the absence or presence of curcumin $(5-40 \mu \mathrm{M})$ for $24 \mathrm{~h}$, and were lysed and analyzed for the expression levels of proteins as indicated. (B) Densitometric analysis of the Bax/Bcl-2 ratio from (A) shows a dose-dependent increase in response to curcumin. Bcl-2, B-cell lymphoma 2; Bax, Bcl-2-associated X protein.
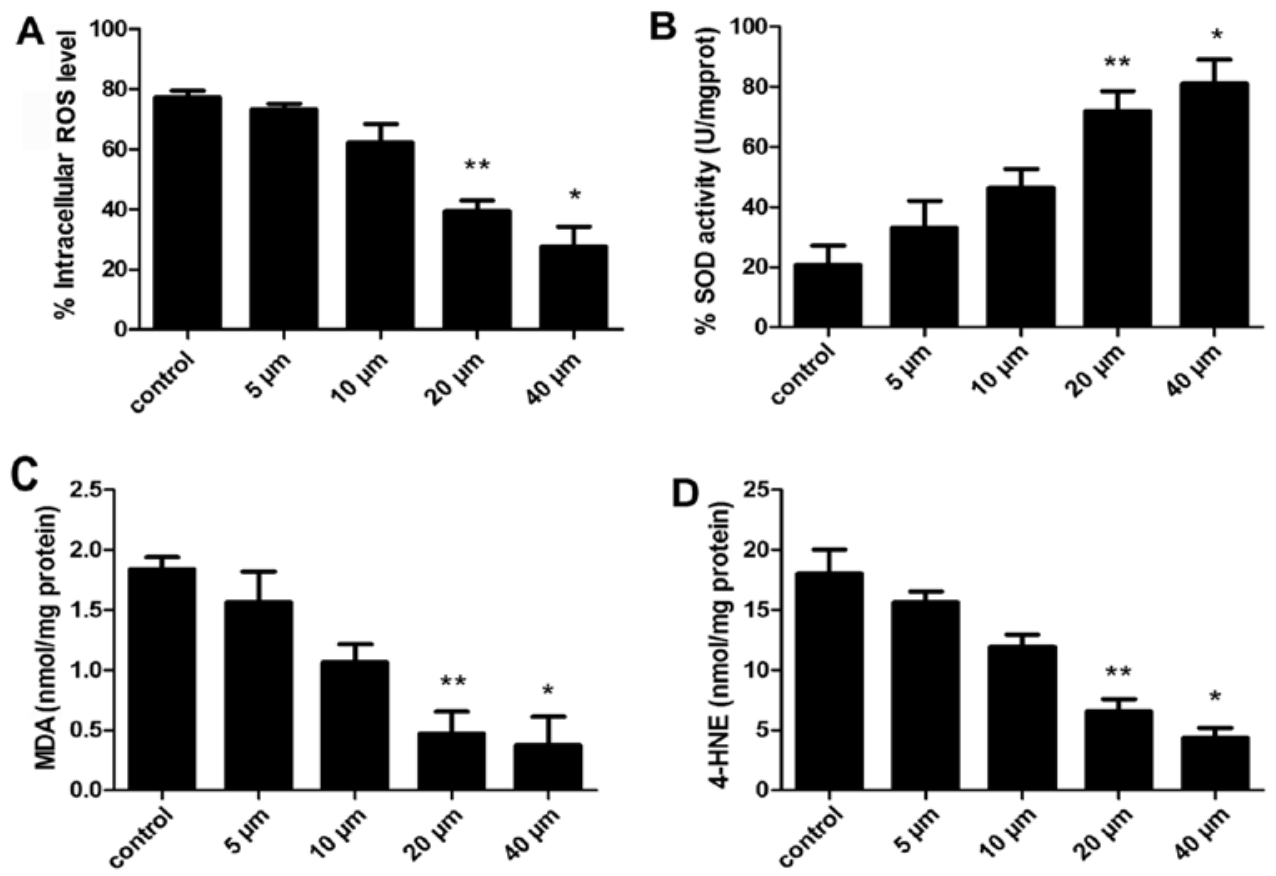

Figure 7. Effects of curcumin on ROS, SOD, MDA and 4-HNE. (A) Curcumin concentrations $>20 \mu \mathrm{M}$ resulted in a decline of ROS levels $\left({ }^{* *} \mathrm{P}<0.05\right.$, ${ }^{*} \mathrm{P}<0.01$ ). (B) SOD activity sharply increased in response to curcumin in a dose-dependent manner. (C) Curcumin treatment for $24 \mathrm{~h}$ decreased MDA levels in A549 cells in a dose-dependent manner $\left.{ }^{(*} \mathrm{P}<0.05,{ }^{,} \mathrm{P}<0.01\right)$. (D) ELISA assay showed increased levels of 4 -HNE after exposure to different doses of curcumin for $24 \mathrm{~h}$. ROS, reactive oxygen species; SOD, superoxide dismutase; MDA, malondialdehyde; 4-HNE, 4-hydroxynonenal.

possible mechanisms by which curcumin induced the apoptosis of A549 cells, cells were exposed to 5-40 $\mu \mathrm{M}$ curcumin and ROS activity was evaluated using a DCF kit. Changes in DCF fluorescence were detected by flow cytometric analysis. The proportion of cells with lower fluorescence intensity was decreased in cells exposed to $20 \mu \mathrm{M}$ curcumin for $24 \mathrm{~h}$ (Fig. 7A), indicating that curcumin significantly decreased the level of ROS in a dose-dependent manner.

Subsequently, the effects of curcumin were examined on SOD levels. As shown in Fig. 7B, exposure of A549 cells to $5-40 \mu \mathrm{M}$ curcumin for $24 \mathrm{~h}$ had a significant increase of intracellular SOD levels compared with the control following 20 and $40 \mu \mathrm{M}$ treatment $(\mathrm{P}<0.05, \mathrm{P}<0.01$, respectively).

To evaluate the role of oxidative stress on mitochondrial function, the effect of lipid peroxidation (MDA and 4-HNE) induced by curcumin in A549 cells was evaluated. After $24 \mathrm{~h}$ of exposure to curcumin, the degree of lipid peroxidation was determined by measuring MDA in cells. As illustrated in Fig. 7C, curcumin treatment reduced MDA levels below baseline values compared with the control cells $(\mathrm{P}<0.05)$.

Another biomarker of oxidative stress, the lipid peroxidation marker 4-HNE $(14,28)$, was examined. As shown in Fig. 7D, A549 cells treated with 5-40 $\mu \mathrm{M}$ curcumin for $24 \mathrm{~h}$ had significantly lower levels of 4 -HNE protein adducts $(\mathrm{P}<0.05)$.

Roles of HSP70 in curcumin-induced cell apoptosis. The conclusion drawn thus far is that curcumin-induced A549 cell apoptosis has a relative association with oxidative stress. To further investigate whether oxidative stress was involved in the curcumin-induced cell death, the level of HSP70 was analyzed by western blot analysis. A549 cells were exposed to various concentrations of curcumin $(5-40 \mu \mathrm{M})$ for $24 \mathrm{~h}$, and the cells were lysed and analyzed for the expression of proteins as indicated. Notably, as shown in Fig. 8, the expression of HSP70 was inhibited in a dose-dependent manner. 

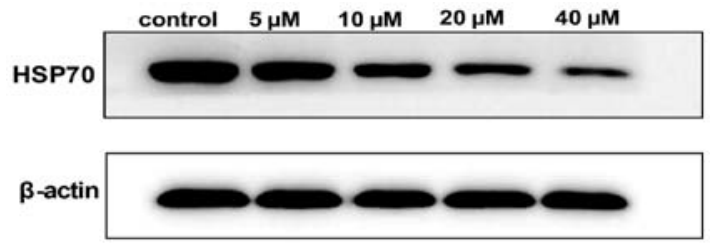

Figure 8. Roles of HSP70 in curcumin-induced cell apoptosis. Cells were treated with curcumin $(0-40 \mu \mathrm{M})$ for $24 \mathrm{~h}$ and HSP70 levels were analyzed by western blotting. HSP70, heat-shock protein 70 .

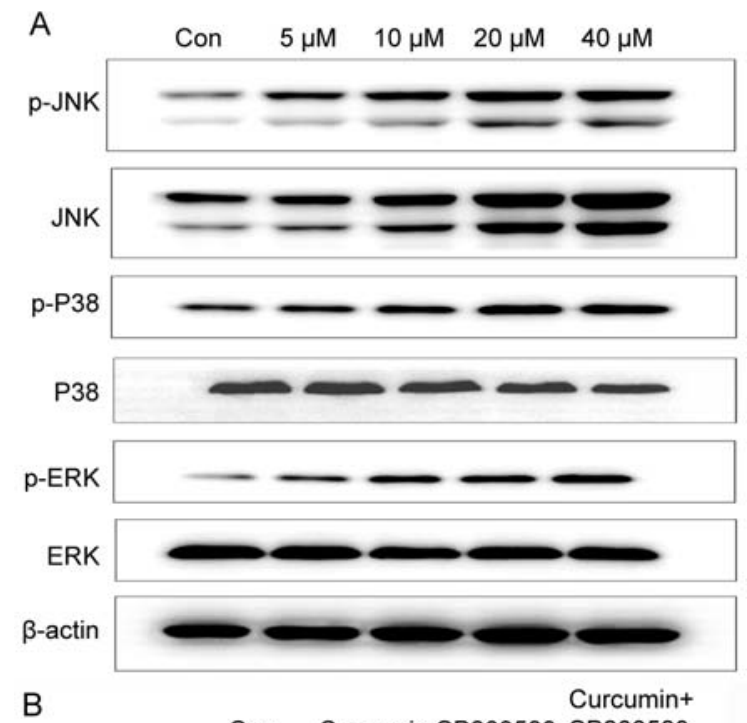

B

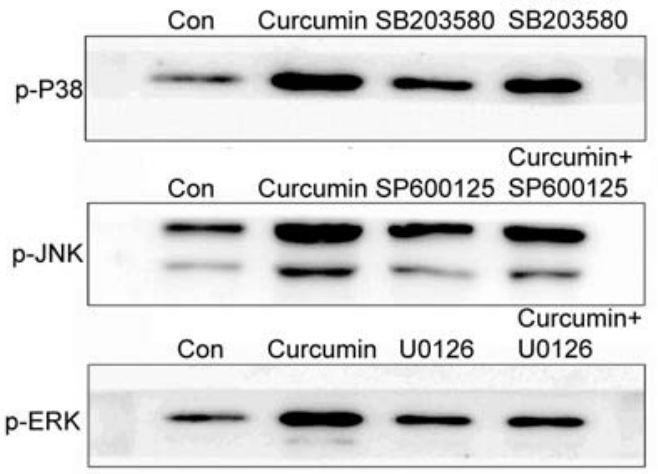

Figure 9. Effect of curcumin on the mitogen-activated protein kinase signaling pathways in A549 cells. (A) Cells were treated with curcumin $(0-40 \mu \mathrm{M})$ for $24 \mathrm{~h}$ and ERK, p38 and JNK steady state and phosphorylated levels were analyzed by western blotting. (B) Cells were treated with $20 \mu \mathrm{M}$ curcumin for $24 \mathrm{~h}$ in the presence or absence of SB203580, U0126 or SP600125. The activations of ERK, p38 and JNK were evaluated by western blot analysis ERK, extracellular signal-regulated kinase; JNK, c-Jun N-terminal kinase.

Role of MAPK signaling in curcumin-induced cell death. MAPK pathways have a vital role in signal transduction of extracellular stimuli to the nucleus and subsequent activation of gene transcription. The MAPK signaling cascade can be activated by oxidative stress, which affects cell proliferation and apoptosis. When the redox balance is disturbed, HSP70 activates JNK phosphorylation, and the MAPK signaling pathway is activated, triggering a series of downstream signal responses. To examine whether curcumin-mediated apoptosis is involved in the MAPK signaling pathways, the levels of total and phosphorylated ERK, p38 and JNK protein were examined in A549 cells treated with 5-40 $\mu \mathrm{M}$ curcumin for $24 \mathrm{~h}$ by western blot analysis. With the increase of concentrations of curcumin, the activation of phosphorylated ERK, JNK and $\mathrm{p} 38$ proteins was also enhanced, whereas, total ERK, p38 and JNK protein had no significant changes (Fig. 9A). These data suggest that the MAPK signaling pathway is involved in curcumin-mediated apoptosis. To investigate whether these kinases were required for curcumin-induced A549 cell apoptosis, the effects of MAPK inhibitors on cellular apoptosis and MAPK activation were examined. Cells were preincubated with U0126 (ERK inhibitor), SB203580 (P38 inhibitor) or SP600125 (JNK inhibitor) for $1 \mathrm{~h}$, and subsequently exposed to $20 \mu \mathrm{M}$ curcumin for $24 \mathrm{~h}$. As shown in Fig. 9B, p38 phosphorylation was reduced in cells pretreated for $1 \mathrm{~h}$ with $20 \mu \mathrm{M}$ SB203580. Similarly, JNK phosphorylation was also reduced in cells pretreated with $20 \mu \mathrm{M}$ SP600125 (Fig. 9B). However, ERK phosphorylation was increased in cells pretreated for $1 \mathrm{~h}$ with $20 \mu \mathrm{M}$ U0126. These results demonstrate that curcumin induces the activation of p38, JNK and ERK, indicating that the MAPK pathway is involved in curcumin-induced apoptosis of A549 cells.

\section{Discussion}

Multiple studies have shown that curcumin promotes antitumor effects by induction of apoptosis $(25,26)$. However, the proapoptotic role of curcumin and underlying mechanism remains unknown. In the present study, curcumin not only inhibited cell proliferation of A549 cells by MTT assay, but also induced apoptosis in a dose- and time-dependent manner. A concentration of $20 \mu \mathrm{M}$ curcumin exhibited significant growth inhibition in A549 cells. As evidence of apoptosis, cells treated with $20 \mu \mathrm{M}$ curcumin showed cell shrinkage with numerous apoptotic bodies compared with untreated cells as observed by electron microscopy.

Two general pathways are involved in apoptosis, including the death receptor-mediated extrinsic and mitochondria-mediated intrinsic pathways (27). The mitochondria-mediated apoptotic pathway is characterized by loss of MMP and release of cytochrome $c$ from mitochondria into the cytoplasm (28). In the present study, the mitochondrial-specific cationic dye, JC-1, was used to confirm the loss of MMP upon exposure to curcumin, and an immunofluorescence assay showed cytochrome $c$ translocation, occurring in a dose-dependent manner. Therefore, curcumin induced the apoptosis of A549 cells through mitochondria-mediated apoptotic pathways.

Oxidative stress is a physiological state in the body, and when the body suffers from a variety of harmful stimulation, highly reactive molecules, such as active oxygen free radicals (ROS) and reactive nitrogen free radicals, are generated in large quantities and the redox balance is broken. These changes induced apoptosis and even tissue damage through the mitochondria signaling pathway, endoplasmic reticulum stress pathway and death receptor pathway (29-31). Oxidative stress is closely associated with cell apoptosis. The physiological state of the ROS level participates in the regulation of cell proliferation: When intracellular ROS levels are above a certain threshold, ROS inhibit the cell cycle, leading to DNA rupture, cell apoptosis and necrosis. A higher state of oxidation is often maintained in the tumor cells, this higher oxidation state activate certain 
transcription factors and associated genes, such as $N F-\kappa B$ and $A P I$, thus, ensuring the survival, proliferation and migration of tumor cells. In the present study, curcumin affected intracellular oxidative stress, decreased ROS and increased SOD activity, further reducing the generation of MDA and 4-HNE (32). These changes trigger the intrinsic apoptotic pathway: HSP70 expression is restrained, the proapoptotic protein Bax and antiapoptotic protein $\mathrm{Bcl}-2$ are activated, MMP is lost, cytochrome $c$ is released into the cytoplasm, the caspase-9/3 classical apoptotic pathway is activated, and eventually cells die $(33,34)$. When cells were pretreated with the HSP70 inhibitor, the indicators of the redox state did not show any change, however, MMP, cytochrome $c$ and the Bax/Bcl-2 ratio were significantly changed, and cells were prevented from undergoing cell apoptosis. Accordingly, oxidative stress is the upstream signal of HSP70, and the Bcl-2 family is the downstream point of HSP70, and subsequently it activates cellular apoptosis via the mitochondrial signaling pathway.

HSPs are a group of proteins that can rapidly respond to any physical changes, including high temperature, metal, drugs, poisoning and oxidative stress (35). HSP70 is one of the most important members of the HSP family, and is closely associated with tumor cell apoptosis (36). HSP70 has a high expression level in tumor cells (particularly malignant tumor cells), and has an important role of antitumor cell apoptosis through participating in the process of gene regulation and immune response. Certain studies have shown that HSP70 is expressed highly under stress conditions, such as heat shock and oxidative stress and subsequently activate the Bc1-2 family protein, compete Apaf-1 with caspase-9, thus, causing the inactivation of caspase-9, which affects the cytoplasm of the caspase cascade and inhibits apoptosis. The study by Stankiewicz et al (37) suggested that HSP70 induced tumor cells apoptosis mainly by blocking heat stress, inhibiting the activity of Bax and protecting the release of apoptotic initiation factors from mitochondria. Therefore, the anti-apoptosis function of HSP70 is indicated by regulation of the $\mathrm{Bcl}-2$ family activity (38). In the present study, curcumin regulated the intracellular redox state to suppress the activity of HSP70, thus, inducing cells apoptosis through activating the mitochondrial apoptotic pathways.

The MAPK signaling pathways are important signal transduction pathways in eukaryotic cells that mediate signal transduction from the cell surface to the nucleus. These pathways are closely associated with cell proliferation, survival, differentiation, apoptosis and other physiological processes (39). There are three groups of MAPK pathways: The ERK1/2 MAPK, P38MAPK and JNK/SAPK MAPK families. The ERK1/2 MAPK family is mainly associated with cell differentiation and proliferation (40). The JNK and P38 family mainly participate in the regulation of cell apoptosis $(41,42)$. Certain studies showed that excess ROS can interfere with a variety of signaling pathways, and all the MAP kinases exhibit sensitivity to ROS and oxidizing agents $(43,44)$. The MAPK signaling cascade can be activated by oxidative stress, which affects cell proliferation and apoptosis. Numerous studies have stated that HSP70 mediated cell apoptosis through inhibition of JNK phosphorylation. When the redox balance is disturbed, HSP70 activates JNK phosphorylation, and the MAPK signaling pathway is activated, triggering a series of downstream signal responses (45). In the present study, phosphorylated JNK and p38 were increased in response to curcumin, whereas ERK was reduced in a dose-dependent manner. The present results showed that curcumin induced the activation of p38, JNK and ERK, and the MAPK signaling pathway was involved in the induction of apoptosis of A549 cells.

In conclusion, the present findings indicated that curcumin inhibited the proliferation of A549 cells by inducing apoptosis in a dose- and time-dependent manner. Additionally, curcumin induced apoptosis in A549 cells via oxidative stress-dependent mitochondria and MAPK signaling pathways. These results suggest the potential of curcumin as a promising candidate for lung cancer therapy.

\section{Acknowledgements}

The present study was supported by a grant from the Laboratory of Cancer Epigenetics, Biomedical Research Center, Sir Run Run Shaw Hospital, School of Medicine, Zhejiang University and Zhejiang Provincial Natural Science Foundation of China (grant no. 64212006). The authors would like to thank the Laboratory of Cancer Epigenetics, Biomedical Research Center, Sir Runrun Shaw Hospital, School of Medicine, Zhejiang University and Zhejiang Provincial Key Laboratory of Gastroenterology for providing the experimental facilities, instruments and guidance.

\section{References}

1. Jemal A, Bray F, Center MM, Ferlay J, Ward E and Forman D: Global cancer statistics. CA Cancer J Clin 61: 69-90, 2011.

2. Ferlay J, Shin HR, Bray F, Forman D, Mathers C and Parkin DM: Estimates of worldwide burden of cancer in 2008: Globocan 2008. Int J Cancer 127: 2893-2917, 2010.

3. Chiang CJ, Chen YC, Chen CJ, You SL and Lai MS; Taiwan Cancer Registry Task Force: Cancer trends in Taiwan. Jpn J Clin Oncol 40: 897-904, 2010.

4. Bray F, Jemal A, Grey N, Ferlay J and Forman D: Global cancer transitions according to the human development index (2008-2030): a population-based study. Lancet Oncol 13: 790-801, 2012.

5. Conforti F and Menichini F: Phenolic compounds from plants as nitric oxide production inhibitors. Curr Med Chem 18: 1137-1145, 2011.

6. Satia JA, Littman A and Slatore CG: Associations of herbal and specialty supplements with lung and colorectal cancer risk in the VITamins and Lifestyle study. Cancer Epidemiol Biomarkers Prev 18: 1419-1428, 2009.

7. Cassileth BR, Deng GE and Gomez JE: Complementary therapies and integrative oncology in lung cancer: ACCP evidence-based clinical practice guidelines (2nd edition). Chest 132 (3 Suppl): 340S-354S, 2007.

8. Guzman-Villanueva D, El-Sherbiny IM, Herrera-Ruiz D and Smyth HD: Design and in vitro evaluation of a new nano-microparticulate system for enhanced aqueous-phase solubility of curcumin. Biomed Res Int 2013: 724763, 2013.

9. Xu P, Yao Y, Guo P, Wang T, Yang B and Zhang Z: Curcumin protects rat heart mitochondria against anoxia-reoxygenation induced oxidative injury. Can J Physiol Pharmacol 91: 715-723, 2013.

10. Chen CC, Sureshbabul M, Chen HW, Lin YS, Lee JY, Hong QS, Yang YC and Yu SL: Curcumin suppresses metastasis via Sp-1, FAK inhibition, and E-cadherin upregulation in colorectal cancer. Evid Based Complement Alternat Med 2013: 541695, 2013.

11. Ahn JC, Kang JW, Shin JI and Chung PS: Combination treatment with photodynamic therapy and curcumin induces mitochondria-dependent apoptosis in AMC-HN3 cells. Int $\mathrm{J}$ Oncol 41: 2184-2190, 2012. 
12. Chen QY, Wu LJ, Wu YQ, Lu GH, Zhan JW, Jiang ZY, Yan J and Zhou JY: Molecular mechanism of trifluoperazine induces apoptosis in human A549 lung adenocarcinoma cell lines. Mol Med Rep 2: 811-817, 2009.

13. Zhang J, Qi HW and Wu CG: Research of anti-proliferation of curcumin on A549 human lung cancer cells and its mechanism. Zhong Yao Cai 27: 923-927, 2004 (In Chinese).

14. Tian DZ, Zhu $\mathrm{H}$ and Liang YJ: Effects and mechanisms of curcuminon apoptosis of lung adenocarcinoma A549 cells. Chin J Clin Pharmacol 15: 8-10, 2006.

15. Chen QY and Wang YY: Curcumin induces apoptosis in human lung adenocarcinoma A549 cells through a reactive oxygen species-dependent mitochondrial signaling pathway. Oncol Rep 23: 397-403, 2010.

16. Li PM, Li YL, Liu B and Wang WJ: Curcumin inhibits MHCC97H liver cancer cells by activating ROS/TLR-4/caspase signaling pathway. Asian Pac J Cancer Prev 5: 2329-2334, 2014.

17. Gopal PK, Paul M and Paul S: Curcumin induces caspase mediated apoptosis in JURKAT cells by disrupting the redox balance. Asian Pac J Cancer Prev 15: 93-100, 2014.

18. Kaushik G, Kaushik T and Yadav SK: Curcumin sensitizes lung adenocarcinoma cells to apoptosis via intracellular redox status mediated pathway. Indian J Exp Biol 50: 853-861, 2012.

19. Fuenzalida K, Quintanilla R, Ramos P, Piderit D, Fuentealba RA, Martinez G, Inestrosa NC and Bronfman M: Peroxisome Proliferator-activated receptor gamma upregulates the Bcl-2 anti-apoptotic protein in neurons and induces mitochondrial stabilization and protection against oxidative stress and apoptosis. J Biol Chem 282: 37006-37010, 2007.

20. Wang XH, Qin Y, Hu MH and Xie Y: Dendritic cells pulsed with hsp70-peptide complexes derived from human hepatocellular carcinoma induce specific anti-tumor immune responses. World J Gastroenterol 11: 5614-5620, 2005.

21. Arya R, Mallik M and Lakhotia SC: Heat shock genes-integating cell survival and death. J Biosci 32: 595-610, 2007.

22. Stankiewicz AR, Lachapelle G, Foo CP, Radicioni SM and Mosser DD: Hsp70 inhibits heat-induced apoptosis upstream of mitochondria by preventing Bax translocation. J Biol Chem 280 : 38729-38739, 2005

23. Mishra S, Kapoor N, Mubarak Ali A, Pardhasaradhi BV Kumari AL, Khar A and Misra K: Differential apoptotic and redoxregulatory activities of curcumin and its derivatives. Free Radic Biol Med 38: 1353-1360, 2005.

24. Skommer J, Wlodkowic D and Pelkonen J: Cellular foundation of curcumin induced apoptosis in follicular lymphoma cell lines. Exp Hematol 34: 463-474, 2006.

25. Zhu DJ, Chen XW, Wang JZ and Ju YL: Proteomic analysis identifies proteins associated with curcumin-enhancing efficacy of irinotecan-induced apoptosis of colorectal cancer LOVO cell. Int J Clin Exp Pathol 7: 1-15, 2013.

26. Shehzad A, Lee J and Lee YS: Curcumin in various cancers. Biofactors 39: 56-68, 2013.

27. Lv ZD, Liu XP and Zhao WJ: Curcumin induces apoptosis in breast cancer cells and inhibits tumor growth in vitro and in vivo. Int J Clin Exp Pathol 7: 2818-2824, 2014.

28. Xue X, Yu JL, Sun DQ, Kong F, Qu XJ, Zou W, Wu J and Wang RM: Curcumin induces apoptosis in SGC-7901 gastric adenocarcinoma cells via regulation of mitochondrial signaling pathways. Asian Pac J Cancer Prev 15: 3987-3992, 2014.

29. Siddique YH, Naz F and Jyoti S: Effect of curcumin on lifespan, activity pattern, oxidative stress, and apoptosis in the brains of transgenic Drosophila model of Parkinson's disease. Biomed Res Int 2014: 606928, 2014. doi:10.1155/2014/606928.
30. Fuenzalida K, Quintanilla R, Ramos P, Piderit D, Fuentealba RA, Martinez G, Inestrosa NC and Bronfman M: Peroxisome proliferator-activated receptor gamma up-regulates the $\mathrm{Bcl}-2$ anti-apoptotic protein in neurons and induces mitochondrial stabilization and protection against oxidative stress and apoptosis. J Biol Chem 282: 37006-37015, 2007.

31. Wall NR, Mohamm RM and AI-Katib AM: Bax:Bcl-2 ratio modulation by bryostatin 1 and novel antitubulin agents is important for susceptibility to drug induced apoptosis in the human early pre-B acute lymphoblastic leukemia cell line, Reh. Leuk Res 23: 881-888, 1999.

32. Su JY, Lai HQ and Chen JP: Natural borneol, a monoterpenoid compound, potentiates selenocystine-induced apoptosis in human hepatocellular carcinoma cells by enhancement of cellular uptake and activation of ROS-mediated DNA damage. PLoS One 8: 635-642, 2013.

33. Heath-Engel HM and Shore GC: Mitochondrial membrane dynamics, cristae remodelling and apoptosis. Biochim Biophys Acta 1763: 549-560, 2006.

34. Kang SK, Cha SH and Jeon HG: Curcumin-induced histone hypoacetylation enhances caspase-3-dependent glioma cell death and neurogenesis of neural progenitor cells. Stem Cells 5: $165-174,2006$.

35. Westerheide SD and Morimoto RI: Heat shock response modulators as therapeutic tools for diseases of protein conformation. J Biol Chem 280: 33097-33100, 2005.

36. Jiang YG, Wang YM and Li QF: Expression significance of HLA-DR antigen and heat shock protein 70 in hepatocellular carcinoma. World J Gastroenterol 9: 1139, 2001.

37. Stankiewicz AR, Livingstone AM, Mohseni N and Mosser DD: Regulation of heat-induced apoptosis by Mcl-1 degradation and its inhibition by Hsp70. Cell Death Differ 16: 638-647, 2009.

38. Schmitt E, Maingret L, Puig PE, Rerole AL, Ghiringhelli F, Hammann A, Solary E, Kroemer G and Garrido C: Heat shock protein 70 neutralization exerts potent antitumor effects in animal models of colon cancer and melanoma. Cancer Res 66: 4191-4197, 2006.

39. Poddar R and Paul S: Novel crosstalk between ERK MAPK and p38 MAPK leads to homocysteine-NMDA receptor mediated neuronal cell death. J Neurochem 124: 558-570, 2013.

40. Chen K, Zhang S, Ji Y, Li J, An P, Ren H, Liang R, Yang J and $\mathrm{Li} \mathrm{Z}$ : Baicalein inhibits the invasion and metastatic capabilities of hepatocellular carcinoma cells via down-regulation of the ERK pathway. PLoS One 8: e72927, 2013.

41. Rane MJ, Song Y, Jin S, Barati MT, Wu R, Kausar H, Tan Y, Wang Y, Zhou G, Klein JB, et al: Interplay between Akt and p38 MAPK pathways in the regulation of renal tubular cell apoptosis associated with diabetic nephropathy. Am J Physiol Renal Physiol 298: F49-F61, 2010.

42. Tarapore RS, Yang Y and Katz JP: Restoring KLF5 in esophageal squamous cell cancer cells activates the JNK pathway leading to apoptosis and reduced cell survival. Neoplasia 15: 472-480, 2013.

43. Son Y, Cheong YK and Kim NH: Mitogen-activated protein kinases and reactive oxygen species: how can ROS activate MAPK pathways? J Signal Transduct 2011: 792639, 2011.

44. Chen Z, Jiang H, Wan Y, Bi C and Yuan Y: $\mathrm{H}_{2} \mathrm{O}_{2}$-induced secretion of tumor necrosis factor- $\alpha$ evokes apoptosis of cardiac myocytes through reactive oxygen species-dependent activation of p38 MAPK. Cytotechnology 64: 65-73, 2012.

45. Ha YJ, Seul HJ and Lee JR: Ligation of CD40 receptor in human B lymphocytes triggers the 5-lipoxygenase pathway to produce reactive oxygen species and activate p38 MAPK. Exp Mol Med 43: 101-110, 2011. 